\title{
NON-TRENCH GUIDED HOLES DRIFTING BY THE STATIC PIN- HOLE METHOD
}

\author{
Thomas Bock*, Alexander Drovnikov**, Sergej Menshenin ${ }^{* *}$, Yuri Popkov**, \\ Irina Bulgakova** \\ * Technical University Munich, Germany, thomas.bock@bri.ar.tum.de \\ ** South Russian State Technical University, Russia i.bulgakowa@gmx.deT

\begin{abstract}
Now there is a requirement in the simple and reliable, universal and effective systems providing given accuracy of conducting of holes. At creation of commercial plants of the directed lining of holes it is necessary to correlate integrally a design of head gear (HG) and installations as a whole, ways and means of an automatic gauging and management. Again created principles and devices of the directed lining of holes of small diameter a method of static puncture should be based on tall controllability of devices and the control of an actual rule (position) executive HG. The constructed control algorithms allow to detour the obstacles, move in straight and curve trajectories with the specified speed, to decrease the rate of construction's movement, to perform orientation of construction and provide its smooth installation into the projective position.
\end{abstract}

Keywords: robotization, construction, control algorithms, visual sensor, mobile robots, planning of the robot's movement, kinematics.

\section{INTRODUCTION}

The control and management are carried out on an actual end result of measurements and are invariant in relation to numerous disturbing affectings. The available information on non-trench to a lining of horizontal technical lines of small diameter not to the full reflects an actual condition of question. Acting norm give only over-all recommendations whenever possible application of ways and methods of obturated passing, that in practice considerably complicates the proved sampling the equipment and production engineering in view of concrete conditions of construction and maintenance of effective and trouble-free manufacture of these works. The executed work provides an opportunity of creation of installations of the directed lining of snake holes with a method of static puncture due to implementation of given accuracy of lining of holes at the least power consumption of process in view of physicomechanical properties of passable soils and fitted for the further automation.

Tool HG or it spacely - orienting handling mechanism (SOHM) directly influence a direction of lining of the hole, therefore research of system «HG - the hole» it is impossible without the analysis of his interactings with passable soil. The directed lining of holes we shall fathom conducting $\mathrm{HG}$ on beforehand projected direction with application artificial and the account of natural bending.

\section{THEORETICAL AND EXPERIMEN- TAL RESEARCHES}

The purpose of the lead researches will consist in raise of efficiency of the directed lining of snake holes by perfection of designs of installations, fitted for the further automation. The object in view is attained by maintenance of necessary position in space of the connected parts of the kinematic couple underlying SOHM, due to an adjustable difference of angular speeds of her parts and developed; a way of the directed lining of holes; control systems and designs HG with the rational parameters proved by mathematical model of interactings in system «soil SOHM - HG - beginning - the monitoring system and managements».

HG represents the handling mechanism which designing is not obviously possible without the kinematic and kinetostatistical the analysis. Structural features HG will consist in necessity of management of him in medium inaccessible to a visual study at conservation of tall extent of positioning and accuracy of improvement of the given coordinates of traffic, and also in restriction of its dimensional parameters (according to production engineering of conducting works and parameters of passable holes). Relative motions of parts of mechanical system depend on an aspect of used kinematic couples. The weakest part of all known methods of development of holes is the monitoring system and managements HG.

Thus, there is a necessity of perfection active, controlled on a command, ways of orientation and development of new ways of management by traffic $\mathrm{HG}$, set up on creation of the developing moment or 
deflecting force, by change of geometry HG. It is offered to carry out an adjustment of direction $\mathrm{HG}$ at the moment of lining of the hole due to maintenance of necessary position in space SOHM by regulating a difference of angular speeds of its connected parts twirled in one or counter directions. Thus the position of target link SOHM is controlled concerning longitudinal axis $\mathrm{HG}$, and a position of an axis of gear - concerning a vertical irrespective of possible turn bore-pipe, being by a gauge length. Processes of bend bore-pipe at its interacting with soil, orientation $\mathrm{HG}$ for formation of holes of the given direction and a profile at a rotary drilling and a puncture are identical and can be observed on uniform physical and mathematical models. The reasons of skew HG in the hole - inhomogeneity of physicomechanical properties of soils, design features bore-pipe and HG, and also lapses at work. Among new development creation of deflecting tools on baseline built the small-sized engine is perspectiv.

With the purpose of implementation of controlled lining of holes a lot of hardware components and controlled HG is developed. However practically all of them are developed for conditions of drilling.

The controlled static puncture is a low-cost, however, low-cost and sweeping way of formation of holes with the least power consumption and having the specific habits: an axial load; absence of twirl bore-pipe which rigidity renders essential effect on process of puncture of the hole; nonstationary medium (inhomogeneity of soils); presence of the big deflecting perturbations.

Demands to developed installations of the directed lining of holes by a method of a static puncture are formulated:

- Again created principles and devices of the directed passing of holes should be based on tall controllability of devices;

- The control and management are carried out on an actual end result of measurements and are invariant in relation to numerous disturbing affectings;

- The control action is formed in view of concrete technology requirements to installation and conditions of its work.

For achievement of tall extent of positioning of target link observed SOHM it should have six extents of mobility: five extents of mobility for removing a target link in required coordinates with a high level of accuracy, and one extent of mobility for moving all mechanism along his own spinning ax. (According to production engineering of accomplishment of works on a static puncture, it is carried out by the power device which has been not connected with SOHM). Alternatives of designs SOHM which target part has four extents of mobility as there is no necessity of destruction of an earth file due to twirl SOHM (is not provided twirl of target link at his arrangement on one axis with the case of the mechanism) are possible.
Raise of functionality SOHM is attained by one of three ways:

- with the help of constructive complications of the mechanism on the basis of kinematic couples 5 class-rooms (in basic rotational);

- application of kinematic couples of the higher class-room;

- on the basis of combinations of constructive complications to application of kinematic couples of the higher class-room.

A)

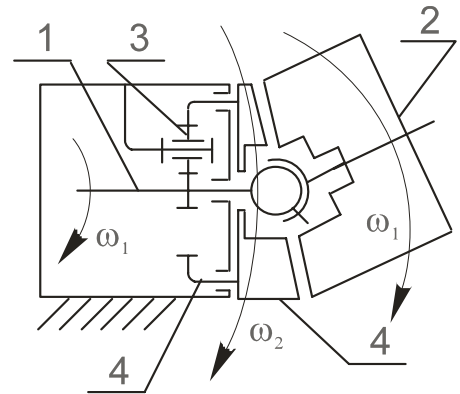

The patent № 2169069

B) $w=6 \times 4-5 \times 3-4 \times 1=5$

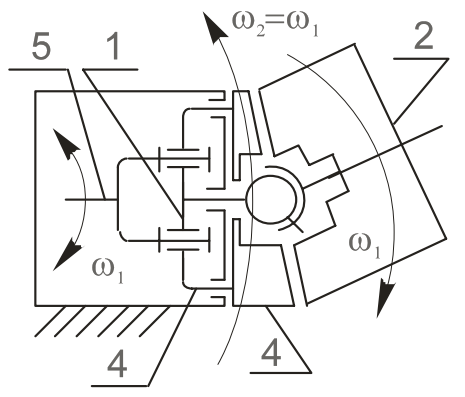

The patent № 2168587 $w=6 \times 4-5 \times 3-4 \times 1=5$

C)

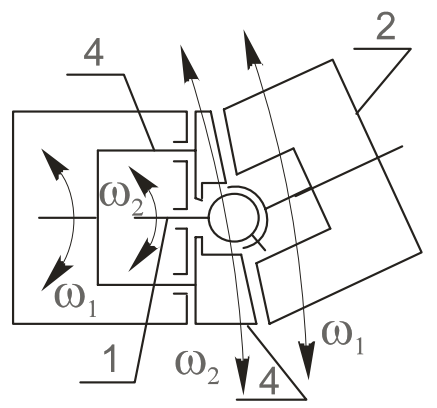

The copyright certificate № 1138317 , The copyright certificate № 905065

$w=5$

Fig. 1

$\mathbf{1}$ - the cardan shaft; 2 - an output link; 3 - a gear; 4 - a deflecting link, joint with socket; 5 - a power shaft, joint with a double free-wheeling clutch; $\omega_{1}-$ an angular velocity of twirl of cardan shaft (power shaft) and joint with it of deflecting link; $\omega_{2}-$ an angular velocity of twirl of an output link 
Now it is the most expedient to use the third way of raise of functionality of joints. The structural synthesis and the kinematic analysis executive SOHM, and also a synthesis of kinematic couples for the given technology requirements on the basis of the analysis of number of extents of their mobility $-\boldsymbol{w}$ provide an opportunity of creation of the synthesized hinged system - SOHM, consisting of the minimal number of kinematic couples of the maximum class-rooms. Sampling the kinematic scheme of the mechanism on baseline of two structural formulae is proved: $w=3+2 x 1=5, w=$ $2+2 x 1=4$. Alternatives of designs of handling systems offered on the basis of a synthesis presented on figure 1.

For definition of control actions on SOHM problems of kinematic for all suggested alternatives SOHM which look as follows are solved: the direct problem of kinematic will consist in definition of coordinates of an apex of an output link on known importance of an angle of rotation of power shaft of the mechanism; the inverse problem of kinematic provides definition of angular motions of power shaft of the mechanism on a preset position of an apex of an output link.

The analytical decision of problems $\{$ tasks $\}$ of kinematics for the mechanism represented on figure $1, A)$, looks as follows:

$$
\begin{aligned}
& \text { At } \beta_{1}>\beta_{2}: \\
& X=-H \sin \left[2 \operatorname{arctg}\left(\operatorname{tg} \alpha \sin \left(\frac{1}{2}\left(\frac{\delta}{n_{1}}-\frac{\delta}{n_{1} n_{2}}\right)\right)\right)\right] \\
& \cdot \cos \left[\frac{1}{2}\left(\frac{\delta}{n_{1}}+\frac{\delta}{n_{1} n_{2}}\right)\right], \\
& Y=-X \operatorname{tg}\left[\frac{1}{2}\left(\frac{\delta}{n_{1}}+\frac{\delta}{n_{1} n_{2}}\right)\right], \\
& Z=H \cos \left[2 \operatorname{arctg}\left(\operatorname{tg} \alpha \sin \left(\frac{1}{2}\left(\frac{\delta}{n_{1}}-\frac{\delta}{n_{1} n_{2}}\right)\right)\right)\right] \\
& \delta=\frac{2 n_{1} n_{2} \operatorname{arctg}\left(\frac{X}{Y}+90^{0}\right)}{n_{2}+1}, \\
& \delta=\frac{2 n_{1} n_{2} \arcsin \left(\frac{\operatorname{tg}(1 / 2 \arccos (Z / H))}{\operatorname{tg} \alpha}\right)}{\operatorname{At} \beta_{1}<\beta_{2}:}
\end{aligned}
$$

$$
\begin{aligned}
& X=H \sin \left[2 \operatorname{arctg}\left(\operatorname{tg} \alpha \sin \left(\frac{1}{2}\left(\frac{\delta}{n_{1} n_{2}}-\frac{\delta}{n_{1}}\right)\right)\right)\right] . \\
& \cdot \cos \left[\frac{1}{2}\left(\frac{\delta}{n_{1}}+\frac{\delta}{n_{1} n_{2}}\right)\right], \\
& Y=-X \operatorname{tg}\left[\frac{1}{2}\left(\frac{\delta}{n_{1}}+\frac{\delta}{n_{1} n_{2}}\right)\right], \\
& Z=H \cos \left[2 \operatorname{arctg}\left(\operatorname{tg} \alpha \sin \left(\frac{1}{2}\left(\frac{\delta}{n_{1} n_{2}}-\frac{\delta}{n_{1}}\right)\right)\right)\right], \\
& \delta=\frac{2 n_{1} n_{2} \operatorname{arctg}\left(\frac{X}{Y}-90^{0}\right)}{n_{2}+1}, \\
& \delta=\frac{2 n_{1} n_{2} \arcsin \left(\frac{\operatorname{tg}(1 / 2 \arccos (Z / H))}{\operatorname{tg} \alpha}\right)}{1-n_{2}} ;
\end{aligned}
$$

Where $\boldsymbol{H}$ - altitude of an output link on its axis of rotation - distance from the centre of jointing up to an apex of an output link;

$\boldsymbol{n}_{1}, \boldsymbol{n}_{2}$ - reduction rates accordingly the first and second reducers;

$\boldsymbol{\delta}$ - an angle of improvement of the drive;

$\boldsymbol{\beta}_{1}$ and $\boldsymbol{\beta}_{2-}-$ meanings of angular motions accordingly output and deflecting links;

$\boldsymbol{\alpha}$ - an angle of chamier of the combined bases of links of mechanism.

Practically similar dependences are received for other mechanisms represented on figure 1 .

Observed SOHM work at small speeds of twirl of links, at interacting to a ground there is a clearing oscillations in transients, in this connection, it is possible to neglect such merit numbers of management, as factor of dynamism (does not exceed 0,5 on oscillograms of pressure at test of an experimental sample of installation), and fixed a time. In the capacity of criterion at sampling parameters the minimal size of linear deviation $\varepsilon$ of targets of an apex of the output link of mechanism from the valid importance is accepted at passage of path during improvement of the drive for realization of these coordinates. The given criterion allows to provide the greatest accuracy of improvement of control action at admissible speed of its realization. The instance of rated path of output link SOHM is reduced on figure 2.

By results of accounts it is possible to make the following outputs:

- Sampling optimum critical bucklings designed SOHM and angular velocities of twirl of their links it is possible to achieve high-scale of controllability of designed design with technologically required accuracy figure of improvement of a mechanical 


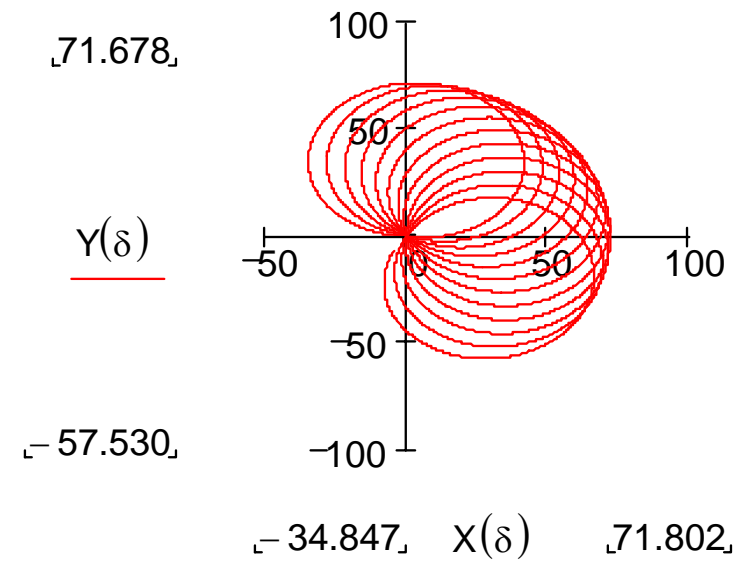

Fig 2.

trajectory, and high-density mechanical trajectories at the minimal size of deviations of the valid path from preset (up to $1,5 \mathrm{~mm}$ ) can be achieved at observance of condition: $n_{1} / n_{2} \approx 1$ oṛ $n_{2} / n_{1} \approx 1$, thus $\boldsymbol{n}_{d v} / \boldsymbol{n}_{1} \boldsymbol{n}_{2} \geq 3,5$ wherẹ $\boldsymbol{n}_{d v}-$ engine speed [turn / minute];

- The designed mechanism is effective and simplis in recustomizing. Offered SOHM meet all demands showed to HG for the directed lining of horizontal boreholes by a method of a static puncture;

- The structural synthesis and the kinematic analysis executive SOHM, and also a synthesis of kinematic pairs for preset technology requirements on the basis of the analysis of number of degrees of their movability ensure an opportunity of
Principle of action of an informational - measuring system - IMS and management HG functionally are carried out as follows

(Figure 4). Position SOHM concerning axis

$\mathrm{HG}$ and a position itself $\mathrm{HG}$ in a race

course of a chink is inspected by four transmitters (inspected sizes are specified in brackets): the displacement pick-up - D1 (a step of introduction $\boldsymbol{L}_{I Z}$ ); a transmitter of a

- The necessary position of handle mechanisms, according to new principles of

space orientation is ensured with a creation of the synthesized hinged system consisting of the minimal number of kinematic pairs of the higher class-rooms; governed difference of angular velocities of conjugate links of the kinematic pair underlying these mechanisms, at the expense of unidirectional or a up-and-down traffic of their links.

Kinematic configurations suggested controlled HG and presented to dependence of capacity of the builtin electric motor on critical bucklings SOHM on figure 3.

vertical - D2 $\left(\boldsymbol{\alpha}_{I Z}\right)$; a transmitter of curving HG - D3 $\left(\boldsymbol{\beta}_{I Z}-\right.$ corner of an inclination (instead of an antiaircraft corner on vertical

chinks), i.e. a corner between a horizontal plane and axis HG and $\boldsymbol{\delta}_{I Z}$ - azimuthal a corner); a transmitter of turnovers of engine $\mathrm{HG}-\boldsymbol{D} 4\left(\boldsymbol{n}_{\boldsymbol{D} V}\right)$. Engineering realization of algorithm of measurement of attitude of axes HG was expressed in development and manufacture of a special transmitter for measurement of an angle of inclination and a bearing angle. After program loading in a

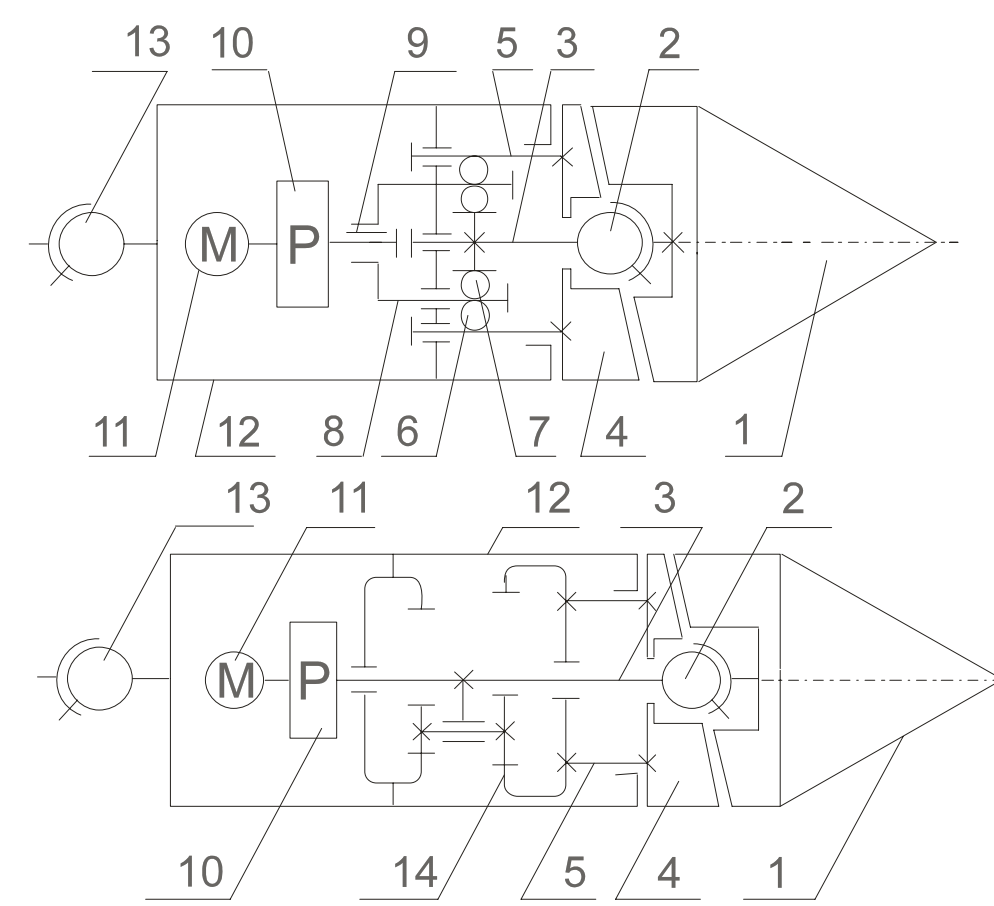

Fig. 3 a)

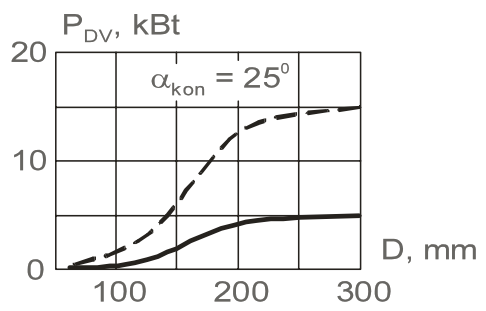

b)

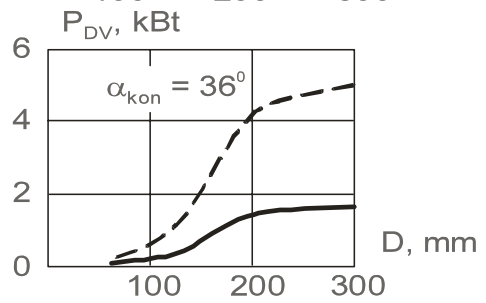

c)

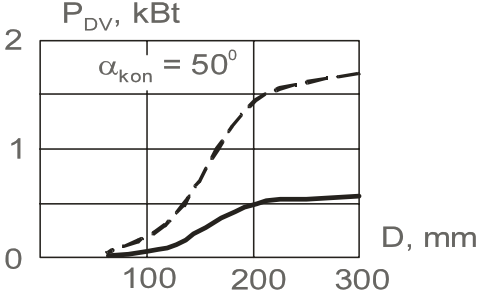

$\mathbf{1}$ - output link SOHM; 2 and 3 - a cardan joint and a shaft; 4 - deflecting link SOHM; 5 - a cartridge clip; 6 and 7 - free-wheeling clutches; 8 - socket; 9 - a keyed joint; 10 - the reducer; 11 - the electric motor; 12 - the case; 13 a lens or a joint; 14 - the second reducer 


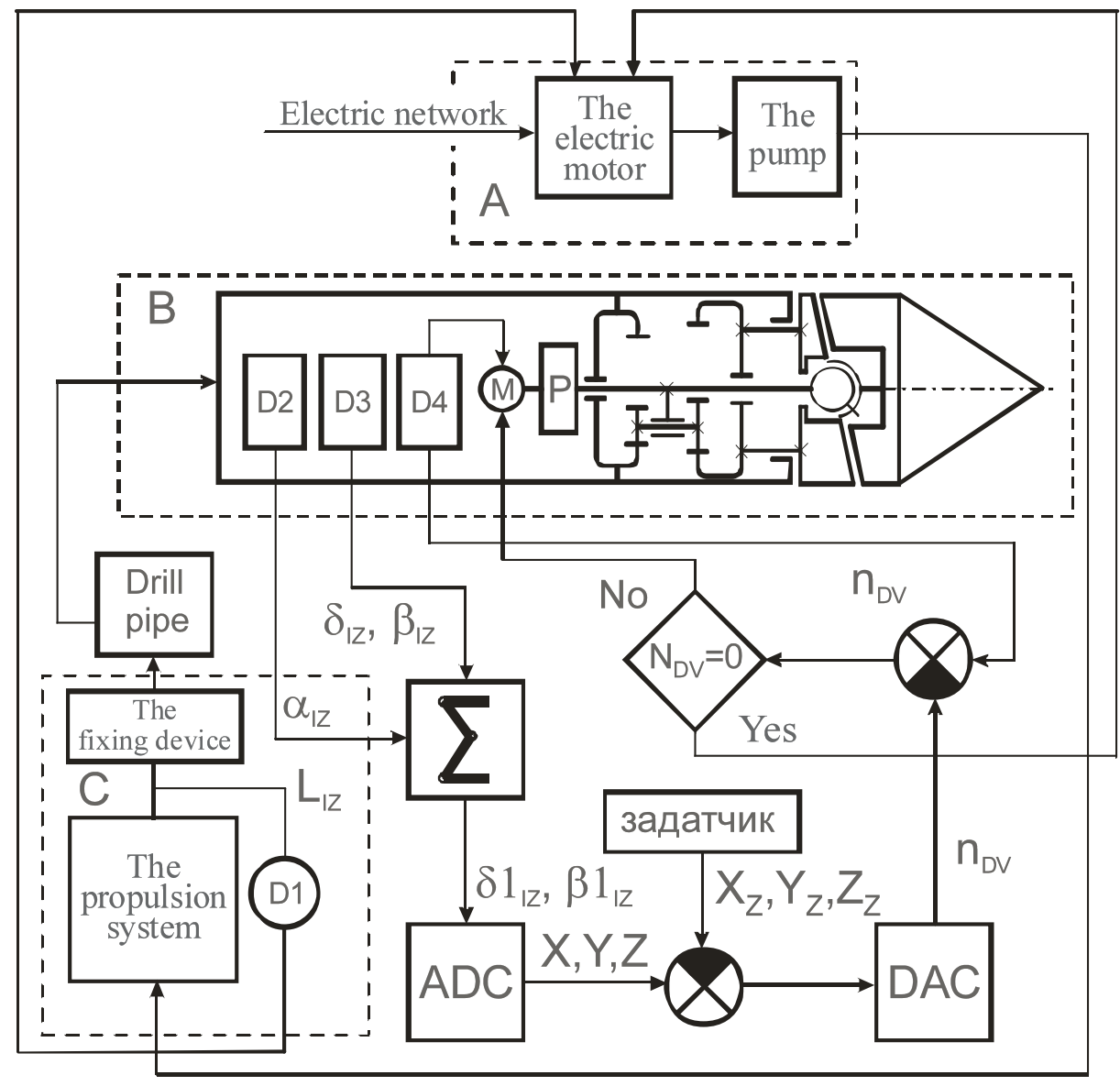

Fig. 4

$\boldsymbol{A}$ - the supply system; $\boldsymbol{B}$ - the head gear; $\boldsymbol{C}$ - the propulsion system; $\boldsymbol{D 1}, \boldsymbol{D} 2, \boldsymbol{D} \mathbf{3}, \boldsymbol{D} \mathbf{4}$ - accordingly: the displacement pick-up, verticals, curving $\mathrm{HG}$, a number of revolutions of the drive; $\boldsymbol{\alpha}_{I Z}, \boldsymbol{\beta}_{I Z}, \boldsymbol{\delta}_{I Z}, \boldsymbol{L}_{I Z}-$ inspected sizes accordingly: a bend angle between sections HG, an angle of inclination, a bearing angle, a step of motion HG; $\boldsymbol{\Sigma}$ - the adder; $\boldsymbol{D} \boldsymbol{A C}$ - analog-converter display; $\boldsymbol{A D C}$ - the analog-digital converter.

computer coordinates of the origin of position $\mathrm{HG}$ are established and original indications of transmitter $\boldsymbol{D} 2$ are removed. The power supplies of the powerplant are carried out from a power room or an off-

line system of power supply. Power cylinders, due to the timbered lock on their stocks, introduce beginning with $\mathrm{HG}$ in hole mouth. The step prosliders is inspected by displacement pick-up $\boldsymbol{D} \mathbf{1}$. A problem of an artificial bending of hole mouth and saving of a preset direction of a lining executes SOHM HG.

At passage HG of a step prosliders $\boldsymbol{L}_{\boldsymbol{I}}$ transmitter $D 1$ gives a signal on the arrangement of switchingoff of the engine of the supply system. Then position HG in a race course of a chink by interrogation of transmitters D2 and D3 is defined. Signals from these transmitters in the impulse form act in integrating element where indications of transmitter $\boldsymbol{D} 3$ - meanings $\boldsymbol{\beta}_{I Z}$ and $\boldsymbol{\delta}_{I Z}$ - are adjusted on size $\boldsymbol{\alpha}_{I Z}$ - indications of transmitter D2. The updated meanings $\beta 1_{I Z}$ and $\delta 1_{I Z}$ act in the analog - digital transducer of an analog-digital converter where obtained datas and constant $\boldsymbol{L}_{I Z}$ will be transformed to coordinates $\boldsymbol{X}, \boldsymbol{Y}, \boldsymbol{Z}$ positions $\mathrm{HG}$. Obtained coordinates are compared with preset $-\boldsymbol{X}_{\boldsymbol{Z}}, \boldsymbol{Y}_{\boldsymbol{Z}}, \boldsymbol{Z}_{\boldsymbol{Z}}-$ the program calculated by a computer proceeding from a prescribed trajectory of a projected line. If targets do not correspond valid on a unbalance signal acting in analog - the converter display $\boldsymbol{D A C}$, is calculated necessary engine speed $\boldsymbol{n}_{\boldsymbol{D} V}$ for reorientation SOHM HG so that on the following step prosliders to return $\mathrm{HG}$ on a preset line of a lining of a chink. Required meaning of size $\boldsymbol{n}_{\boldsymbol{D} V}$ is compared to the valid meaning interrogation of transmitter D4. If the difference of rated and valid sizes $\boldsymbol{n}_{\boldsymbol{D} V}$ is equal to zero, on the electric motor the signal about turning on of the powerplant and the beginning of passage of the following step prosliders acts. At deriving in a logic network of a unbalance signal $-\boldsymbol{n}_{\boldsymbol{D} V} \neq \boldsymbol{0}$ electric motor HG reinstalls it SOHM up to a required position, thus the quantity of turnovers of the engine is inspected by transmitter $D 4$ before observance of condition $\boldsymbol{n}_{\boldsymbol{D} V}=\boldsymbol{0}$. On execution of this operation the signal in the supply system about the beginning of the following step prosliders moves. Obtained indications of transmitters and calculated coordinates of position HG on each step are remembered by a computer, and under these indications the valid line of a chink is under construction.

Experimental researches were carried out in some stages: researches of interactings in system «the instrument - beginning - a ground», researches of process of interacting of the instrument with a ground and tests expirience sample HG containing a built-in control gear and a monitoring system of a position. 
In the capacity of plants for holding researches fullscale installations were used, and tests were carried out in actual conditions. As a result of tests it is established:

- Efficiency of agency of critical bucklings HG on a curvature of a path of a chink and a degree of controllability;

- Necessity of management HG for a lining of the directed chinks, for the lack of an opportunity of deriving of rectilinear chinks in the extent more than 8 meters owing to heterogeneity of transited grounds and habits of a natural bending;

- The Opportunity of creation controlled HG and realizations of the accepted way of an adjustment of a direction of its movement.

- Experimental researches have allowed to define dependence of axial effort $-\boldsymbol{P}_{\boldsymbol{O S}}=\mathrm{f}\left(\boldsymbol{H}, \boldsymbol{V}_{\boldsymbol{G} \boldsymbol{R}}\right)$ for various geometrical parameters of tips in view of physicomechanical characteristics of a ground. $\boldsymbol{V}_{\boldsymbol{G} \boldsymbol{R}}$ at introduction of a tip it was defined as a difference of volume of a section of a drill pipe in length, equal current length of the paved chink, and volume of the tip introduced into soil - target link SOHM and a drill pipe.

The constructed control algorithms allow to detour the obstacles, move in straight and curve trajectories with the specified speed, to decrease the rate of construction's movement, to perform orientation of construction and provide its smooth installation into the projective position.

\section{REFERENCES}

[1] Melikhov A.N., Berstein L.S., Korovin S.Ya. Blurred Condition Models of DecisionMaking.-Taganrog: TRTI-96.

[2] Lozano-Perez T. Spacial planning: a configuration space approach // IEEE Transactions on Computers, 1983, pp.C-32:108-120.

[3] Lebedev D.V. Application of Wave Propagation Neuron Network for Planning of Safe Paths of Plane Robots // Information Technologies, № 10, 2000, pp.19-26.

[4] Lebedev D.V. Neuronetwork Model for Paths Planning of Autonomous Robot, taking into account Dynamic Changes in the environment // Intelligent Systems, № 6, 2002, pp.195-214. 